\title{
Fast, accurate and non-empirical determination of the lattice thermal conductivities of I-III-VI 2 chalcopyrite semiconductors
}

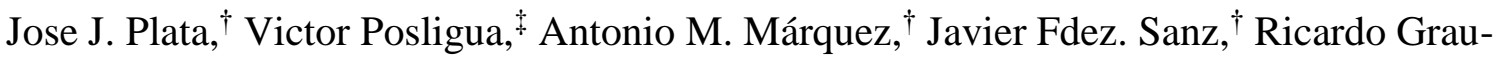 \\ Crespo ** $^{*}$ \\ †Departamento de Química Física, Facultad de Química, Universidad de Sevilla, Seville, Spain. \\ "Department of Chemistry, University of Reading, Whiteknights, Reading RG6 6DX, UK. \\ *Email: r.grau-crespo@reading.ac.uk
}

\begin{abstract}
The use of computer simulation to predict the lattice thermal conductivity of materials has the potential to accelerate the discovery of new thermoelectric materials. However, the accurate prediction of this property from first principles, without input from experiment, is very computationally demanding, which limits the use of high-throughput strategies in thermoelectric materials design. We present here an accurate, fast, and non-empirical determination of the lattice thermal conductivities of a large family of semiconductors, with composition $A B X_{2}$ (I-III-VI 2 ), with $A=\mathrm{Cu}, \mathrm{Ag} ; B=\mathrm{Al}, \mathrm{Ga}$, In, Tl; and $X=\mathrm{S}$, Se, Te. We solve the Boltzmann transport equation with force constants derived from density functional theory calculations and machine-learning-based regression algorithms, reducing between one and two orders of magnitude the computational cost with respect to conventional approaches of the same accuracy. The results are in good agreement with available experimental data and allow us to rationalize the role of chemical composition, temperature and nanostructuring on the thermal conductivities across this important family of semiconductors.
\end{abstract}

KEYWORDS: chalcopyrite, lattice thermal conductivity, thermoelectric materials, machine learning.

\section{INTRODUCTION}

The thermal conductivity $(\kappa)$ of semiconductors is a property of key importance for their applications in thermoelectric, photovoltaic, and electronic devices [1-3]. The computational prediction of this property has the potential to accelerate the discovery and design of materials 
for such applications [4-6]. In semiconductors and insulators, the dominant contribution to the thermal conductivity is phonon transport, which is responsible for the so-called lattice thermal conductivity. One of the most accurate methods for obtaining lattice thermal conductivities from computer simulations is based on Boltzmann's transport equation (BTE) for phonons [7], which requires the calculation of the second-order and, at least, third-order interatomic force constants (IFCs). Traditionally, the IFCs are predicted by calculating atomic forces in supercells where one or two atoms are systematically displaced from their equilibrium positions, using density functional theory (DFT) $[8,9]$. The number of displacements, and therefore of DFT calculations, required to obtain third order IFCs is usually very high, and thus this step represents the bottleneck in the prediction of $\kappa$ from first principles [5, 8-11]. Alternatively, empirical expressions can be applied to efficiently estimate $\kappa$, without using IFCs [4, 12-15]. However, these methods rely on experimental data that are not always available and suffer from accuracy issues that make them unsuitable when quantitative information is required, such as for the calculation of the figure of merit of thermoelectrics.

In recent years there has been progress in new algorithms to accelerate the calculation of IFCs, for example, using techniques such as compressive sensing and machine learning (ML) to extract the necessary information from a much smaller number of DFT calculations [16, 17]. These new methods open the door to the accurate calculation of $\kappa$ for large families of materials and are the ideal tool for exploring the chemical and physical behavior that govern this property. Because of the sensitivity of $\kappa$ to synthetic conditions, which control grain size and defect chemistry, any trends in thermal conductivities extracted from experiments under different conditions should be taken very cautiously. In contrast, computer modelling allows a direct comparison of intrinsic thermal conductivity behavior across compositions and temperatures.

We present here an investigation of the thermal conductivity of chalcopyrite semiconductors, based on the full BTE solution with IFCs obtained from DFT via ML-based regression algorithms. We consider 20 experimentally reported compositions of I-III- $\mathrm{VI}_{2}$ chalcopyrite-structured non-magnetic semiconductors, as listed in Ref. [18]. They correspond to all $A B X_{2}$ compositions with $A=\mathrm{Cu}, \mathrm{Ag} ; B=\mathrm{Al}, \mathrm{Ga}$, In; and $X=\mathrm{S}$, Se, Te, plus two compositions with $B=\mathrm{Tl}\left(\mathrm{CuTlS}_{2}\right.$ and $\left.\mathrm{CuTlSe}_{2}\right)$. The chalcopyrite structure, with tetrahedrally coordinated $A(\mathrm{I})$ and $B(\mathrm{III})$ cations (Figure 1), is a versatile structure for semiconducting materials with rich chemistry and electronic behavior (they can be obtained both $p$ - and $n$-type) and unexpensive synthesis. They have attracted research and commercial interest for a wide range of applications, including thermoelectric [19-21] and photovoltaic [22-24] devices. The 
thermal conductivity behavior is important for these applications; therefore a systematic understanding of phonon transport in these materials across compositions and temperatures, and of the effect of nanostructuring on the thermal conductivity, is important for the design of better device components.

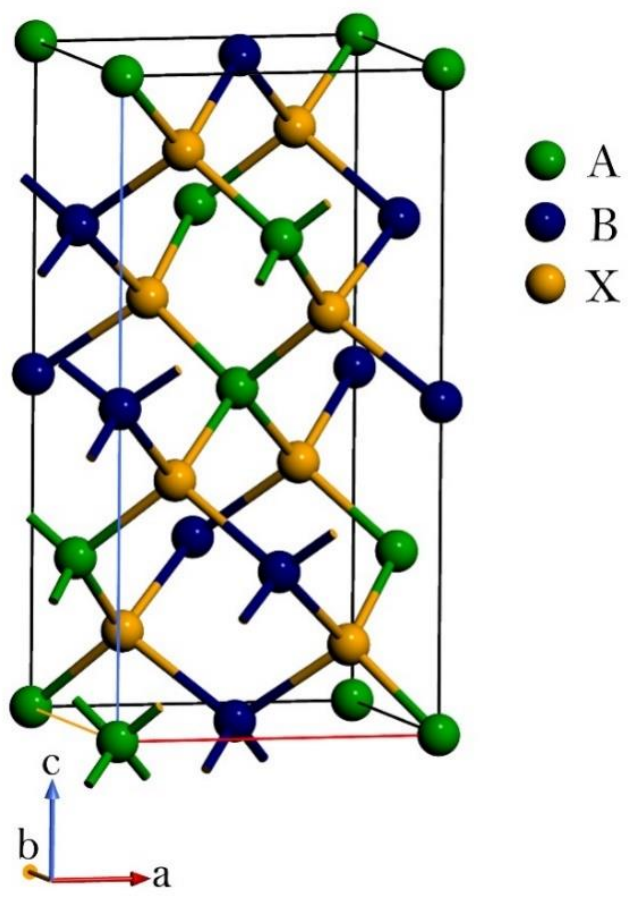

Figure 1. Tetragonal unit cell of the chalcopyrite structure with composition $A B X_{2}$.

\section{METHODOLOGY}

DFT calculations. Chalcopyrite conventional cells (16 atoms) were fully relaxed using the VASP code [25] with the projector-augmented wave (PAW) method [26]. Energies were obtained using the generalized gradient approximation (GGA) proposed by Perdew et al. combined with Grimme's D3 van der Waals corrections [27] and a plane-wave basis set with a cutoff of $500 \mathrm{eV}$. The number of valence electrons for each atom was selected following standards proposed by Calderon et al. [28]. Reciprocal space integrations were performed using a k-point mesh of $8 \times 8 \times 4$ for the unit cell, and commensurate grids for the supercells. Structures were considered fully relaxed when forces acting over each atom were smaller than $10^{-7} \mathrm{eV} / \mathrm{A}$. An additional support grid for the evaluation of the augmentation charges was included to reduce the noise in the forces. The forces required for obtaining the IFCs were calculated using a $4 \times 4 \times 2$ supercell, as recently used by Park et al. for $\mathrm{CuFeS}_{2}$ [29]. 
Force constants prediction and machine learning. We used the machine-learning-based approach implemented in the HiPhive package [17], to extract second-, third-, and fourth-order force constants within optimized cutoff distances. Although the fourth-order force constants do not enter in our BTE model, their inclusion leads to a better regression for the force constant potential (FCP) model. A training set of forces was obtained from DFT calculations for a number of structures with random displacements of all atoms, generated via a Monte Carlo algorithm that penalizes displacements producing too short interatomic distances. The average displacement amplitude for each configuration was $\sim 0.12 \AA$. The force constants were then determined from multi-linear regression to the DFT forces with the recursive feature elimination (RFE) algorithm, which only keeps the most important features (force constants) during the FCP model construction. Reducing the number of parameters via the RFE algorithm is justified because one can expect that only some interaction terms are relevant in the FCP. This ML algorithm leads to a less complex and more transferable model and to faster convergence of the IFCs with the number of structures employed.

The convergence of the parameters (number of structures and cutoff distances) involved in the FCP model was tested by assessing the variation of the force errors, the phonon frequencies, and the lattice thermal conductivity itself. We used $\mathrm{CuGaTe}_{2}$ as a representative case for these tests because of the availability of experimental data for that composition. The comparison with $\kappa$ values obtained from the more computationally demanding "full-DFT" approach is also presented in the case of $\mathrm{CuGeTe}_{2}$. For this composition, cutoff distances of $11 \AA, 6.2 \AA$ and $4 \AA$ for the second, third and fourth-order force constants were found to be sufficient for convergence, and these cutoffs were extrapolated to other compositions based on coordination shells (rather than distances) for consistency. We developed a wrapper code for the HiPhive program that automates the distorted supercell creation, forces calculation using VASP, and the construction of the ML FCPs, which is available for public use [30].

Boltzmann's transport equation solution. Once the FCP model is built, lattice thermal conductivities were obtained by solving the Boltzmann transport equation using the ShengBTE code [9]. We used the full iterative procedure to go beyond the relaxation time approximation. Scattering times were computed including isotopic and three-phonon scattering. A Gaussian smearing of $0.1 \mathrm{eV}$ and a dense mesh of $20 \times 20 \times 10 \boldsymbol{q}$-points were used in all the calculations, balancing the memory demand and the convergence of $\kappa$ with the number of $\boldsymbol{q}$-points. The effects of including non-analytical contributions (NACs) on $\kappa$ were tested in two compounds $\left(\mathrm{CuGaS}_{2}\right.$ and $\left.\mathrm{AgGaS}_{2}\right)$ and only small changes (below 2.5\%) were found, so the results reported 
below do not included NACs, to avoid the extra cost of computing Born effective charges. The scalar values reported correspond to one third of the trace of the thermal conductivity tensor; small anisotropic effects will be discussed below. In what follows, we refer to the calculated lattice thermal conductivity as $\kappa$, which we compare with the experimental total (lattice + electronic) values, because the electronic contribution can be expected to be very small.

\section{RESULTS AND DISCUSSION}

The lattice parameters of all compounds are well reproduced by the calculations, as shown in Figure 2. The mean absolute errors are $0.025 \AA$ (average $0.42 \%$ deviation) for $a$, and $0.170 \AA$ $(1.5 \%)$ for $c$, which are well below the differences in cell parameters across compositions, leading to the good correlations observed in Figure 2. Within this family of compositions, the nature of the anion has the strongest effect on the cell parameters, which tellurides having the largest and sulfides the smallest cell parameters.
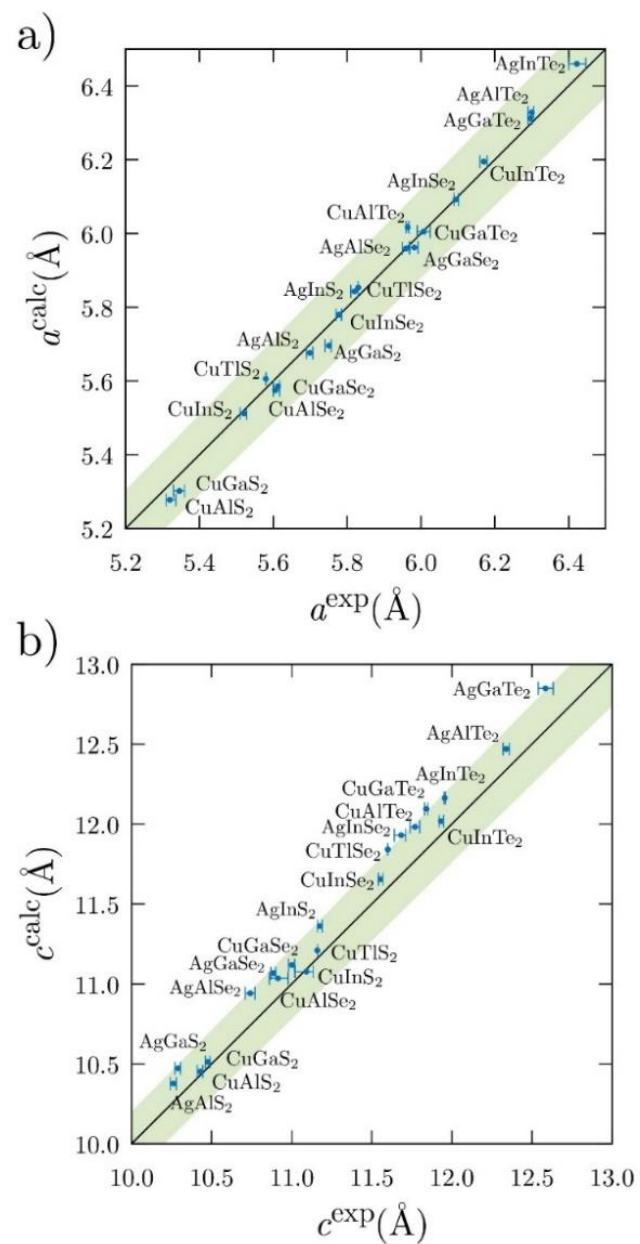

Figure 2. Comparison of DFT-calculated vs experimental cell parameters [18]. The solid line in each plot represents perfect agreement, and the green shaded area represents deviations of up to $2 \%$ from experiment in either direction. 
In agreement with experiment, the nature of the A cation affects the $a$ and $c$ parameters differently, with the $c / a$ ratio being significantly lower for Ag-based (average $c / a=1.86$ ) than for the $\mathrm{Cu}$-based (average $c / a=1.98$ ) chalcopyrites. This has been observed elsewhere and related to the fact that the $\mathrm{Ag}-X-\mathrm{Ag}$ bonds are softer than the $\mathrm{Cu}-X-\mathrm{Cu}$ bonds [23]. The deviation from $c / a=2$ characterizes the structural anisotropy in the system. Therefore, we can expect that Ag-based chalcopyrites will have slightly more anisotropic properties than $\mathrm{Cu}$ based ones, which will be confirmed in terms of thermal conductivities below.

Figure 3 illustrates that the force-constant potentials obtained from the ML algorithms in HiPhive give very similar results to those obtained with the more computationally expensive "full-DFT" method, where force constants are determined from DFT calculations for all symmetrically distinct displacements of individual atoms. Using $\mathrm{CuGaTe}_{2}$ as example, Figure 3a gives the dispersion curves obtained by the two methods, which are practically indistinguishable from each other, demonstrating the equivalence between the two sets of second-order force constants.

The third-order force constants are also very close: the calculated lattice thermal conductivity becomes closer to the full-DFT result when the number of structures used in the ML fitting of the forces increases, and they get within 10\% of each other when 16-20 structures are used (Figure $\mathbf{3 b}$ ). This comparison was made using a relatively short cutoff $r_{3 r d}^{\text {cut }}=4.7 \AA$ for the third-order force constants, in such a way that the full-DFT result could be obtained, as this already required the DFT calculation of 348 structures (i.e. 20 times more DFT calculations than when using the ML-based method). This huge saving of computing effort allows the computation of lattice thermal conductivities for the whole family of compounds in a high-throughput fashion.

Furthermore, using the ML-based method we can now afford to increase the third-order cutoff, which leads to a much better agreement between the fitted forces and the DFT forces: e.g. for $\mathrm{CuGaTe}_{2}$, the root mean square error (RMSE) of the fitted forces went below $0.01 \mathrm{eV} / \AA$ with $r_{3 r d}^{\text {cut }}=6.2 \AA$ (Figure 3c). Increasing the third-order cutoff from $4.7 \AA$ to $6.2 \AA$, also affects the calculated lattice thermal conductivity, reducing its value from $12.3 \mathrm{Wm}^{-1} \mathrm{~K}^{-1}$ to 11.8 $\mathrm{Wm}^{-1} \mathrm{~K}^{-1}$ when using forces from 18 structures for fitting, bringing the result slightly closer to the experimental value $\left(10.7 \mathrm{Wm}^{-1} \mathrm{~K}^{-1}\right)$ measured by Bodnar et al. for $\mathrm{CuGaTe}_{2}$ single crystals [31]. The possibility of using larger cutoffs constitutes another advantage of the MLbased method over the full-DFT approach, which is necessarily constrained to small cutoffs 
due to the huge computational cost involved (e.g. over 600 structures would be needed for $\mathrm{CuGaTe}_{2}$ when $r_{3 r d}^{\text {cut }}=6.2 \AA$ ). We have given here the cutoff distances used for $\mathrm{CuGaTe}_{2}$, but it should be noted that the final cutoffs were kept consistent across different compositions by defining them by the coordination shells rather than by distance.

a)

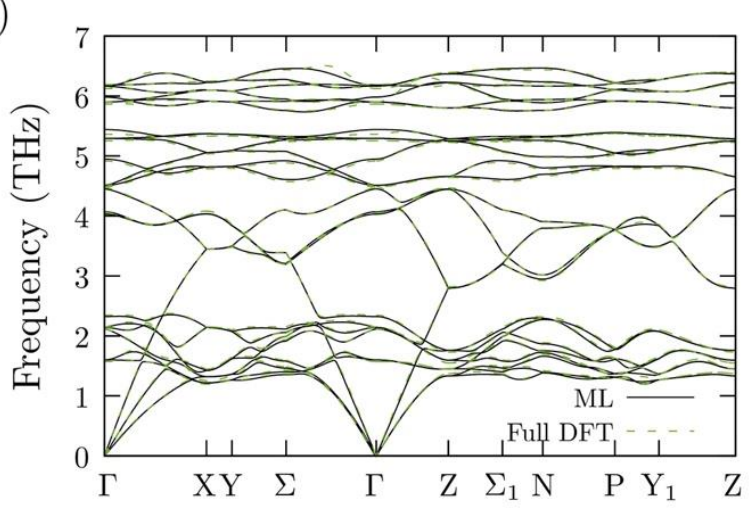

b)

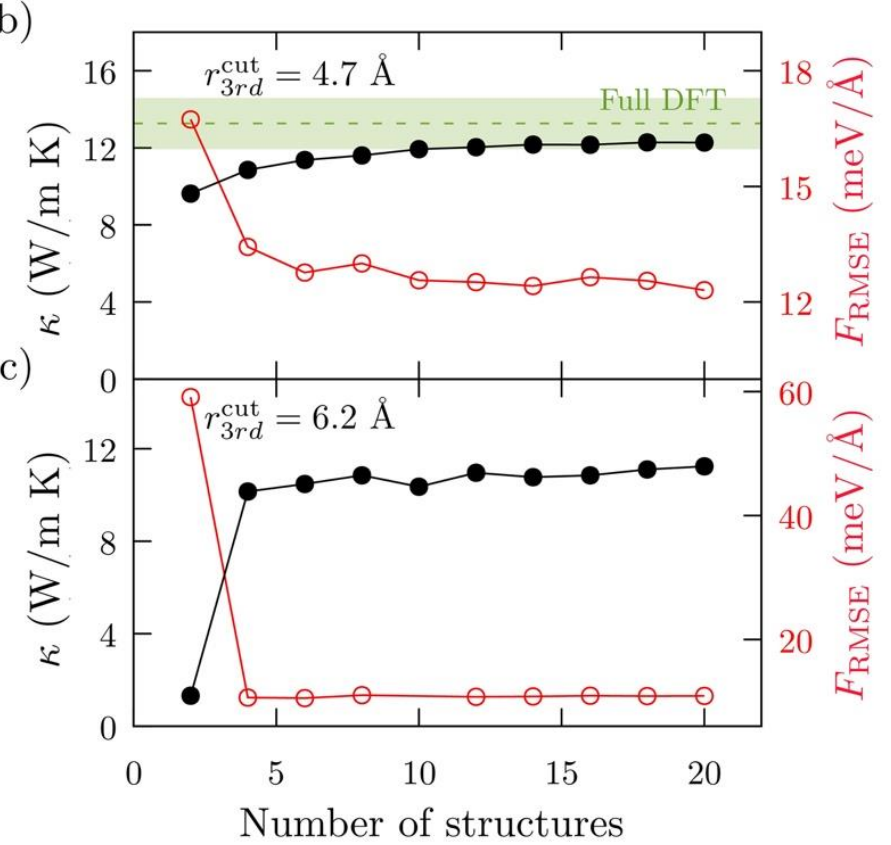

Figure 3. a) Comparison of $\mathrm{CuGaTe}_{2}$ dispersion curves obtained using the machine-learned force constant potential (ML) via HiPhive, with those from the full DFT method via Phonopy. b) Convergence of $\kappa$ with the number of structures used to obtain force constants (with a low third-order cutoff), in comparison with the full-DFT limit. The shaded area represents deviations of up to $10 \%$ in either direction; c) Convergence of $\kappa$ with the number of structures but for a higher third-order cutoff. In $b$ ), c) the points in red represent the evolution of the root mean square error (RMSE) in fitting the forces. 
The calculated average lattice thermal conductivities for all the chalcopyrite compositions are summarized in Table 1. We will first focus on discussing the room-temperature bulk values before examining the effect of temperature and/or nanostructuring. The most obvious trend is that Ag-based chalcopyrites have much lower lattice thermal conductivity than $\mathrm{Cu}$-based ones. The average of the 9 compositions corresponding to $B=\mathrm{Al}$, Ga or In, and $X=\mathrm{S}, \mathrm{Se}, \mathrm{Te}$, is $\sim 6.5$ times higher for $\mathrm{Cu}$-based $\left(9.8 \mathrm{~W} \mathrm{~m}^{-1} \mathrm{~K}^{-1}\right)$ than for Ag-based $\left(1.5 \mathrm{~W} \mathrm{~m}^{-1} \mathrm{~K}^{-1}\right)$ compositions.

Table 1. Calculated lattice thermal conductivities ( $\kappa$ ) for 20 chalcopyrite compositions at $300 \mathrm{~K}$ and $700 \mathrm{~K}$. $L_{0.5}$ is the mean free path cutoff (and therefore approximate nanostructure size) that leads to halving $\kappa$ with respect to the bulk value.

\begin{tabular}{|c|c|c|c|c|}
\hline & \multicolumn{2}{|c|}{$300 \mathrm{~K}$} & \multicolumn{2}{|c|}{$700 \mathrm{~K}$} \\
\hline & $\begin{array}{c}\kappa \\
\left(\mathrm{W} \mathrm{m}^{-1} \mathrm{~K}^{-1}\right)\end{array}$ & $\begin{array}{l}L_{0.5} \\
(\mathrm{~nm})\end{array}$ & $\begin{array}{c}\kappa \\
\left(\mathrm{W} \mathrm{m}^{-1} \mathrm{~K}^{-1}\right)\end{array}$ & $\begin{array}{l}L_{0.5} \\
(\mathrm{~nm})\end{array}$ \\
\hline $\mathrm{CuAlS}_{2}$ & 8.27 & 335 & 3.39 & 159 \\
\hline $\mathrm{CuAlSe}_{2}$ & 7.25 & 159 & 3.06 & 63 \\
\hline $\mathrm{CuAlTe}_{2}$ & 10.2 & 192 & 4.34 & 76 \\
\hline $\mathrm{CuGaS}_{2}$ & 15.6 & 278 & 6.33 & 110 \\
\hline $\mathrm{CuGaSe}_{2}$ & 9.16 & 192 & 3.86 & 76 \\
\hline $\mathrm{CuGaTe}_{2}$ & 11.8 & 192 & 5.03 & 91 \\
\hline $\mathrm{CuInS}_{2}$ & 12.3 & 175 & 5.05 & 76 \\
\hline $\mathrm{CuInSe}_{2}$ & 6.85 & 132 & 2.88 & 63 \\
\hline $\mathrm{CuInTe}_{2}$ & 6.70 & 101 & 2.85 & 52 \\
\hline $\mathrm{CuTlS}_{2}$ & 5.12 & 76 & 2.12 & 52 \\
\hline $\mathrm{CuTlSe}_{2}$ & 2.52 & 43 & 1.07 & 17 \\
\hline $\mathrm{AgAlS}_{2}$ & 2.03 & 43 & 0.86 & 17 \\
\hline $\mathrm{AgAlSe}_{2}$ & 0.94 & 12 & 0.41 & 5 \\
\hline $\mathrm{AgAlTe}_{2}$ & 1.46 & 43 & 0.63 & 17 \\
\hline $\mathrm{AgGaS}_{2}$ & 2.23 & 63 & 0.95 & 25 \\
\hline $\mathrm{AgGaSe}_{2}$ & 0.77 & 6 & 0.33 & 3 \\
\hline $\mathrm{AgGaTe}_{2}$ & 1.43 & 43 & 0.61 & 17 \\
\hline $\mathrm{AgInS}_{2}$ & 2.05 & 30 & 0.88 & 12 \\
\hline $\mathrm{AgInSe}_{2}$ & 0.89 & 10 & 0.38 & 4 \\
\hline $\mathrm{AgInTe}_{2}$ & 1.69 & 110 & 0.72 & 43 \\
\hline
\end{tabular}

To examine the origin of this distinction, in Figures $\mathbf{4 a}$ and $\mathbf{4 b}$ we have plotted the phonon density of states corresponding to $\mathrm{CuGaTe}_{2}$ and $\mathrm{AgGaTe}_{2}$, including the contributions from the 
A metal atoms. Clearly, Ag contribute modes at low frequencies, below $1 \mathrm{THz}$, which do not happen in the $\mathrm{Cu}$ compounds. While the group velocity distribution is similar for both compounds (figure 4c), the low-lying optical modes introduced by Ag lead to higher scattering rates $\left(W_{\text {anh }}\right)$ at those frequencies, which dominate the behavior of the thermal conductivity (Figure 4d).

a)
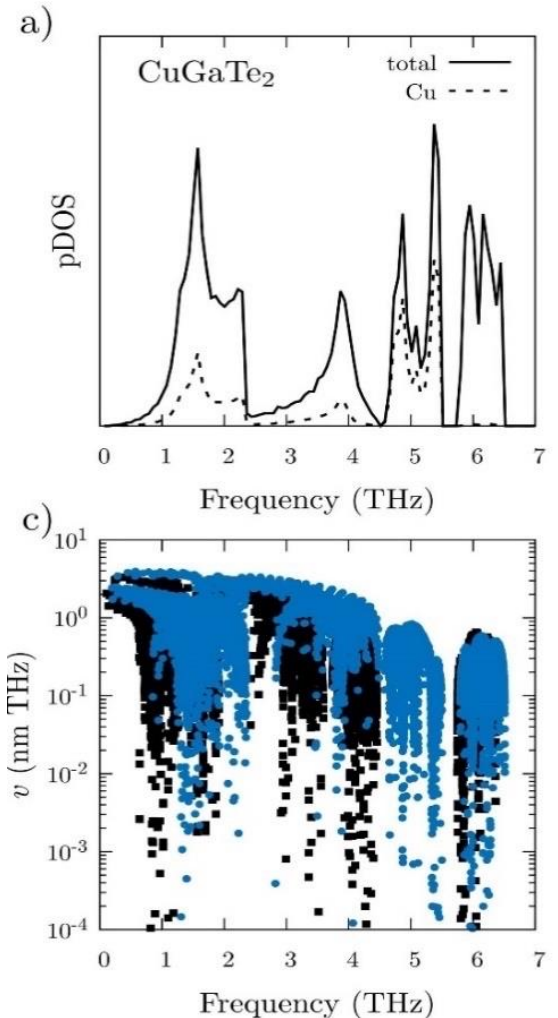

b)

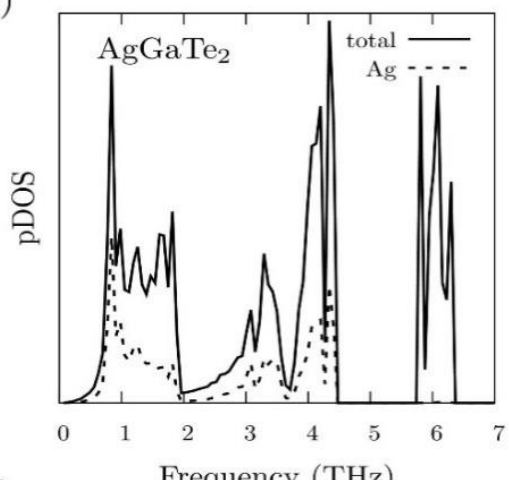

d)

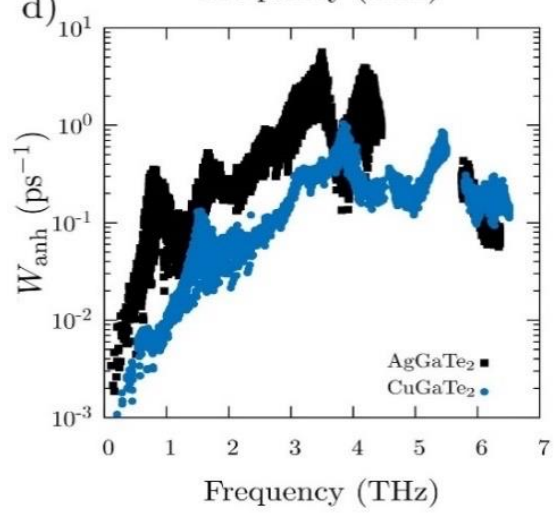

Figure 4. Phonon density of states (pDOS) for a) $\mathrm{CuGaTe}_{2}$ and b) $\mathrm{AgGaTe}_{2}$, showing the projections on the A metal atoms; c) Group velocities vs mode frequency; and d) scattering rates vs mode frequency for the same two compounds.

On the other hand, the trend with the nature of the chalcogen $X$ atom is not monotonous down the group: selenides exhibit lower thermal conductivity than the corresponding sulfides and tellurides. This is illustrated in Figure 5a, by showing that the lattice thermal conductivities of $\mathrm{Cu} B X_{2}$ and $\operatorname{Ag} B X_{2}$ (averaged over $B=\mathrm{Al}, \mathrm{Ga}, \mathrm{In}$ ) reach minimum values when $X=\mathrm{Se}$. This behavior is reminiscent of the non-monotonous variation of the bandgap of lead chalcogenides ( $\mathrm{PbSe}$ has a lower bandgap than both $\mathrm{PbS}$ and $\mathrm{PbTe}$ ), which results from the delicate balance of several electronic factors [32]. The three Ag-based selenide chalcopyrites ( $\mathrm{AgAlSe}_{2}$, $\mathrm{AgGaSe}_{2}$ and $\mathrm{AgInSe}_{2}$ ) are then predicted to have remarkably low lattice thermal 
conductivities, all below $1 \mathrm{~W} \mathrm{~m}^{-1} \mathrm{~K}^{-1}$. The very low thermal conductivity of $\mathrm{AgAlSe}_{2}$ has been recently observed experimentally, and rationalized in terms of an "avoided crossing" feature and low-lying optical modes in the phonon dispersion [33].

The effect of the $B^{3+}$ cation is less regular. For example, making $B=\mathrm{Ga}$ leads to the lowest $\kappa$ among the $\mathrm{Ag}$-based selenides $\operatorname{Ag} B \mathrm{Se}_{2}\left(0.77 \mathrm{~W} \mathrm{~m}^{-1} \mathrm{~K}^{-1}\right.$ at $300 \mathrm{~K}$, which is also the lowest value obtained in this study), but also leads to the composition with the highest $\kappa$ among the Ag-based sulfides, $\operatorname{Ag} B S_{2}$. In the Cu-based compounds, $B=\mathrm{Ga}$ always leads to the highest $\kappa$ across the series of sulfides, selenides and tellurides. This does not mean that $\kappa$ is insensitive to the nature of the $B^{3+}$ cation, just that trends cannot be generalized as in the case of the ions in the $A$ and $X$ sites. In fact, we have also calculated two Cu-based compounds with $B=\mathrm{Tl}$ $\left(\mathrm{CuTlS}_{2}\right.$ and $\mathrm{CuTlSe}_{2}$; other combinations including $\mathrm{Tl}$ were not considered as they do not seem to be stable or there is very little experimental information about them), and they have much lower thermal conductivities than the $\mathrm{Cu} B \mathrm{~S}_{2}$ and $\mathrm{Cu} B \mathrm{Se}_{2}$ compounds with $B=\mathrm{Al}, \mathrm{Ga}$, In. In particular, $\mathrm{CuTlSe}_{2}$ has a $\kappa$ value of $2.52 \mathrm{~W} \mathrm{~m}^{-1} \mathrm{~K}^{-1}$, which is the lowest among the Cu-based chalcopyrites, and comparable with the Ag-based ones.

The analysis above is based on average values along all directions (from the tensor trace). However, the calculation gives the full $\kappa$ tensor, so the slightly anisotropic behavior, due to the tetragonal symmetry of the structure, can be discussed. We pointed out above that $\mathrm{Ag}$ compounds present a larger structural anisotropy than $\mathrm{Cu}$ compounds, with a more pronounced deviation from the ratio $c / a=2$. This is reflected in the anisotropy found in the thermal conductivity: the average $\kappa_{\mathrm{z}} / \kappa_{\mathrm{x}, \mathrm{y}}$ ratio for $\mathrm{Cu}$-based compounds $(0.91)$ is closer to one than for the Ag-based compounds (0.84).

Figures $\mathbf{5 b}$ and $\mathbf{5 c}$ summarize the comparison of our results, and of previous theoretical results, with room-temperature experimental measurements. Clearly, the calculations reported in our work offer the closest agreement so far with experimental data across the family of chalcopyrite semiconductors. This is not surprising as we have employed a more sophisticated model for the evaluation of $\kappa$, compared to previous work. For example, the results reported by Rincon et al. [34] have the highest mean absolute error (MAE), since their calculations were based on a very simple (and computationally inexpensive) analytical model, using a modified version of Leibfried and Schlömann's equation [35] for $\kappa$ as a function of the Debye temperature and the Grüneisen parameter, which were obtained either directly from available experimental data or via extrapolation. Their model widely overestimates the thermal conductivities of the Ag-based chalcopyrites. 

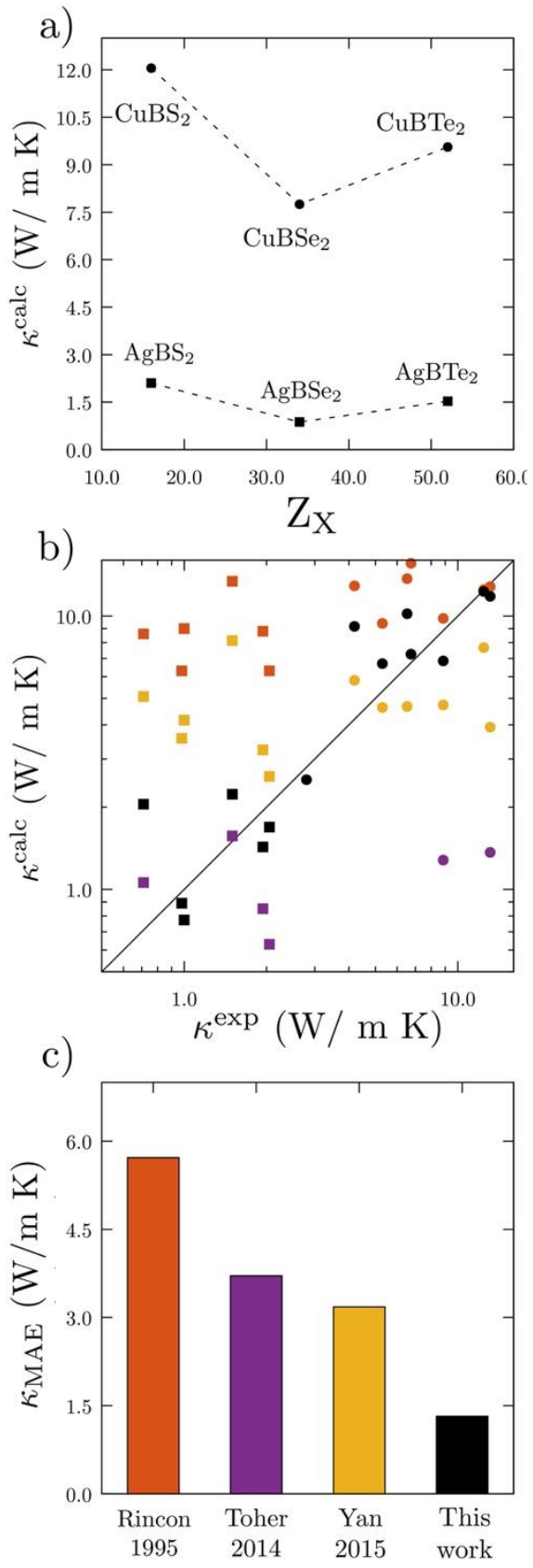

Figure 5. a) Variation of the room-temperature average lattice thermal conductivities of $\mathrm{CuBX}_{2}$ and $\mathrm{AgBX}_{2}$ (over $\mathrm{B}=\mathrm{Al}, \mathrm{Ga}, \mathrm{In}$ ) with the nature of the chalcogen atom $\mathrm{X}=\mathrm{S}, \mathrm{Se}, \mathrm{Te}\left(\mathrm{Z}_{\mathrm{X}}\right.$ are the atomic numbers); b) Comparison of the room-temperature $\kappa$ values calculated in this work and in previous theoretical work, with available experimental data. Black symbols (squares for Ag-based and circles for $\mathrm{Cu}$-based systems) represent the results from this work, whereas symbols of other colors represent previous theoretical determinations; c) Mean absolute errors in this work compared to those in previous theoretical determinations. The colors of the bars match the color of the symbols in (b) for each previous theoretical work reference (Rincon 1995 is Ref. [34]; Toher 2014 is Ref. [6]; Yan 2015 is Ref. [15]).

Experimental data from Refs [20, 21, 36-44]. 
More recently, Toher et al. [6] used an approach not requiring any experimental data. Thermal conductivity was calculated by combining the Slack equation [45] with the Debye temperature and Grüneisen parameter, which were both obtained from DFT calculations by using a quasi-harmonic Debye model. The lack of experimental parameters and the low computational cost make this method suitable for application to a large set of materials; however, it severely underestimates the thermal conductivity of the $\mathrm{Cu}$-based chalcopyrites. Finally, Yan et al. [15] developed an approach based on the Debye-Callaway model [46], which is predictive within one order of magnitude across a large range of experimental data. Although this approach improves results over those obtained from Slack's equation, it required the fitting of parameters from experimental data. Still, the method tends to overestimate the thermal conductivities of the Ag-based chalcopyrites, and underestimate it for the $\mathrm{Cu}$-based chalcopyrites, as shown in Figure 5b. Not only does the approach presented in this work improves the accuracy of the calculation (our MAE is less than half of that from the best previous theoretical work), but it also does so without relying on experimental data, and at a very low computational cost compared with the traditional DFT-based approach to obtain the force constants needed to solve the Boltzmann's transport equation.

We can also compare the predicted evolution of $\kappa$ with temperature with available experimental data. Figure 6a shows typical variations of $\kappa$ with temperature $T$, using $\mathrm{CuInSe} 2$ and $\mathrm{AgInSe} 2$ as examples. The lattice thermal conductivity is dominated by phonon-phonon Umklapp scattering and therefore exhibits a $T^{-1}$ dependence, which reflects the increasing number of phonons available for scattering when temperature increases. The theoretical and experimental curves are fitting using the expression:

$$
\kappa=\kappa_{0}+\frac{m}{T}
$$

where the parameter $m$ characterizes the rate of decreases of the thermal conductivity with temperature. Figure $\mathbf{5 b}$ shows that our calculation gives a reasonable prediction of $m$ values in comparison with experiment. Part of the discrepancy between theoretical and experimental values stem from the presence of other scattering mechanisms such as grain boundaries or fourth-order scattering in the actual compounds, which can modify the $T^{-1}$ relationship [47, 48]. The thermal conductivities of Ag-based chalcopyrites, which are already relatively low, decrease more slowly with temperature than those of the $\mathrm{Cu}$-based chalcopyrites. 

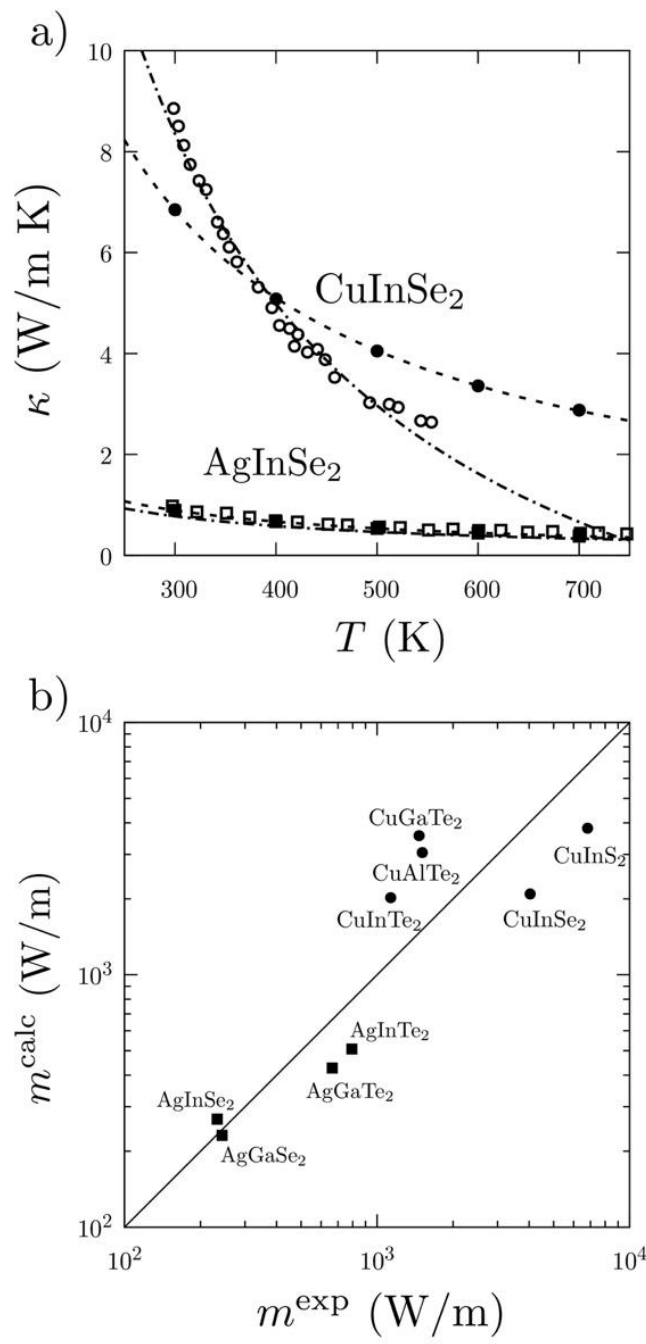

Figure 6. a) Calculated temperature variation of $\kappa$ for representative $\mathrm{Cu}$-based and $\mathrm{Ag}$-based chalcopyrite semiconductors (solid symbols), in comparison with experiment (open symbols; Ref. [49] for $\mathrm{CuInSe}_{2}$ and Ref. [21] for $\mathrm{AgInSe}_{2}$ ); b) calculated temperature variation coefficients in comparison with available experimental data (Ref. [50] for $\mathrm{AgGaSe}_{2}$, Ref. [21] for $\mathrm{AgInSe}_{2}$, Refs. [19, 41, 51] for $\mathrm{AgGaTe}_{2}$, Refs. [21, 41] for $\mathrm{AgInTe}_{2}$, Ref. [42] for $\mathrm{CuInTe}_{2}$, Ref. [49] for $\mathrm{CuInSe}_{2}$, Ref. [42] for $\mathrm{CuAlTe}_{2}$, Ref. [31] for $\mathrm{CuGaTe}_{2}$ and Ref. [49] for $\mathrm{CuInS}_{2}$ ). 

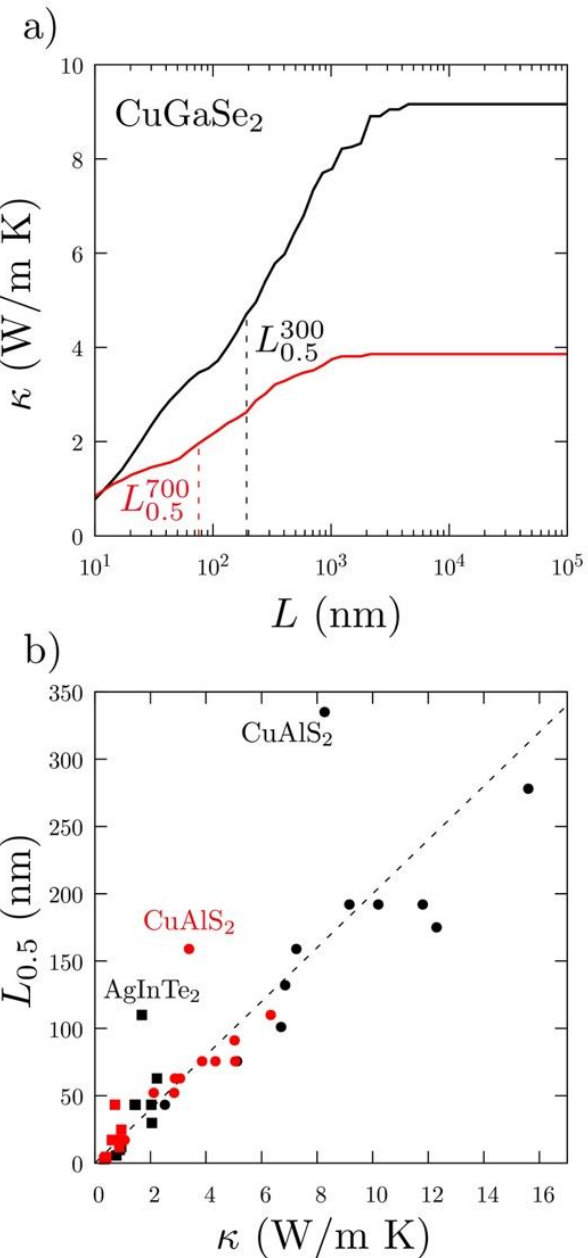

Figure 7: a) Cumulative lattice thermal conductivity from mean-free-path contributions up to distance $L$ for a $\mathrm{Cu}$-based chalcopyrite, indicating the effect that nanostructuring would have on their thermal conductivity. Black lines: $T=300 \mathrm{~K}$; red lines: $T=700 \mathrm{~K}$. b) Correlation between $\mathrm{L}_{0.5}$ and $\kappa$, including points at $300 \mathrm{~K}$ and $700 \mathrm{~K}$. The solid line corresponds to a proportionality constant of $20 \mathrm{~nm} / \mathrm{W} \mathrm{m}^{-1} \mathrm{~K}^{-1}$ between $L_{0.5}$ and $\kappa$. The $\mathrm{CuAlS}_{2}$ and $\mathrm{AgInTe} \mathrm{e}_{2}$ outliers are labelled.

Finally, we discuss the effect of nanostructuring on the thermal conductivities, based on the cumulative value of $\kappa$ up to a given mean free path value. We find that the long mean-freepaths contributions are large for the $\mathrm{Cu}$-based chalcopyrites. For example, for $\mathrm{CuGaSe}_{2}$, particle sizes in the order of $\mu \mathrm{m}$ already have a significant effect on the thermal conductivity (figure 7a). However, the size effect is much weaker in the low- $\mathrm{Ag}$-based chalcopyrites, which therefore do not benefit as much from nanostructuring.

In order to quantify the pattern of $\kappa$ reduction with nanostructuring at a given temperature, we have calculated the particle size (or more exactly, the mean-free-path threshold) that leads to halving the bulk value (figure 7a). The values, that we call $L_{0.5}$, are listed in Table $\mathbf{1}$. We observe that the lower the bulk value of $\kappa$, the smaller the $L_{0.5}$ (figure $7 \mathbf{b}$ ). In fact, a useful rule 
of thumb emerges from that plot: the particle size that is needed to reduce the thermal conductivity by half is roughly $20 \mathrm{~nm}$ per $\mathrm{W} \mathrm{m}^{-1} \mathrm{~K}^{-1}$ of the bulk value of $\kappa$, and the proportionality is not significantly affected by temperature. There are some outliers, notably $\mathrm{CuAlS}_{2}$ and $\mathrm{AgInTe}_{2}$, which are well above the regression line. These are, in fact, interesting cases, because they represent compositions where nanostructuring leads to much faster reduction of the thermal conductivity than for the average chalcopyrite, which is useful for thermoelectric applications.

\section{CONCLUSIONS}

A combination of density functional theory simulations and machine learning regression algorithms have allowed us to efficiently and accurately calculate force constant potentials, and then lattice thermal conductivities, for a large set of semiconductors with the chalcopyrite structure. The computational cost linked to the calculations of the force constants was more than one order of magnitude lower compared to traditional approaches, which are based on systematic atomic displacements. These calculations lead to a number of important insights about the behavior of the thermal conductivity of I-III-VI $\mathrm{VI}_{2}$ chalcopyrite as a function of chemical composition, temperature, and microstructure.

First, we have demonstrated that Ag-based chalcopyrites present considerably lower values of thermal conductivity than $\mathrm{Cu}$-based chalcopyrites, and that this is mainly due to the lower frequencies of the vibrational modes in which Ag atoms participate. These vibrational modes overlap more effectively with acoustic modes, increasing the scattering processes and reducing the scattering times. While no clear trends are found when $B$ cation is substituted, the trend with the nature of the chalcogen $X$ atom is not monotonous down the group: selenides exhibit lower thermal conductivity than the corresponding sulfides and tellurides. Moderate anisotropy is found for $\kappa$, being larger in Ag-based chalcopyrites than in Cu-based chalcopyrites, thus mirroring the structural anisotropy given by the cla ratio. Roomtemperature lattice thermal conductivities are accurately predicted, and the dependence of $\kappa$ on temperature is also in good agreement with experimental data. Finally, the effects of grain size on $\kappa$ have been explored by calculating the cumulative $\kappa$ value up to a certain phonon mean free path. We showed that the particle size needed to reduce the thermal conductivity by half is roughly $20 \mathrm{~nm}$ per $\mathrm{W} \mathrm{m}^{-1} \mathrm{~K}^{-1}$ of the bulk value of $\kappa$, at all temperatures of interest. This relationship provides a useful rule of thumb to facilitate the design of nano-structuring 
strategies to reduce thermal conductivity within this important family of semiconducting materials.

\section{ASSOCIATED CONTENT}

Input and output files from our simulations are available at the online repository: https://doi.org/10.5281/zenodo.5510656. Our wrapper code for HiPhive can be downloaded from https://github.com/NewMaterialsLab/hiPhive_wrapper_NML.

\section{ACKNOWLEDGMENTS}

This work was funded by the Ministerio de Ciencia e Innovación (PID2019-106871GB-I00). JJP, AMC and JFS thankfully acknowledge the computer resources at Lusitania and the technical support provided by Cénits-COMPUTAEX and Red Española de Supercomputación, RES (QS-2021-1-0027, QHS-2021-2-0022). VP and RGC are grateful to the UK Materials and Molecular Modelling Hub for computational resources (Young facility), which is partially funded by EPSRC (EP/P020194/1 and EP/T022213/1).

\section{REFERENCES}

1. Zebarjadi, M., et al., Perspectives on thermoelectrics: from fundamentals to device applications. Energy \& Environmental Science, 2012. 5(1): p. 5147-5162.

2. Yeh, L.-T., R.C. Chu, and W.S. Janna, Thermal Management of Microelectronic Equipment: Heat Transfer Theory, Analysis Methods, and Design Practices. ASME Press Book Series on Electronic Packaging. Applied Mechanics Reviews, 2003. 56(3): p. B46-B48.

3. Cahill, D.G., et al., Nanoscale thermal transport. II. 2003-2012. Applied Physics Reviews, 2014. 1(1): p. 011305.

4. Nath, P., et al., High throughput combinatorial method for fast and robust prediction of lattice thermal conductivity. Scripta Materialia, 2017. 129: p. 88-93.

5. Plata, J.J., et al., An efficient and accurate framework for calculating lattice thermal conductivity of solids: AFLOW-AAPL Automatic Anharmonic Phonon Library. npj Computational Materials, 2017. 3(1): p. 45. 
6. Toher, C., et al., High-throughput computational screening of thermal conductivity, Debye temperature, and Grüneisen parameter using a quasiharmonic Debye model. Physical Review B, 2014. 90(17): p. 174107.

7. Ziman, J.M., Electrons and Phonons: The Theory of Transport Phenomena in Solids. Oxford Classic Texts in the Physical Sciences. 2001, Oxford: Oxford University Press. 568.

8. Togo, A., L. Chaput, and I. Tanaka, Distributions of phonon lifetimes in Brillouin zones. Physical Review B, 2015. 91(9): p. 094306.

9. Li, W., et al., ShengBTE: A solver of the Boltzmann transport equation for phonons. Computer Physics Communications, 2014. 185(6): p. 1747-1758.

10. Chernatynskiy, A. and S.R. Phillpot, Phonon Transport Simulator (PhonTS). Computer Physics Communications, 2015. 192: p. 196-204.

11. Tadano, T., Y. Gohda, and S. Tsuneyuki, Anharmonic force constants extracted from first-principles molecular dynamics: applications to heat transfer simulations. Journal of Physics: Condensed Matter, 2014. 26(22): p. 225402.

12. Toher, C., et al., Combining the AFLOW GIBBS and elastic libraries to efficiently and robustly screen thermomechanical properties of solids. Physical Review Materials, 2017. 1(1): p. 015401.

13. Bjerg, L., B.B. Iversen, and G.K.H. Madsen, Modeling the thermal conductivities of the zinc antimonides ZnSb and Zn ${ }_{4} \mathrm{Sb}_{3}$. Physical Review B, 2014. 89(2): p. 024304.

14. Toberer, E.S., A. Zevalkink, and G.J. Snyder, Phonon engineering through crystal chemistry. Journal of Materials Chemistry, 2011. 21(40): p. 15843-15852.

15. Yan, J., et al., Material descriptors for predicting thermoelectric performance. Energy \& Environmental Science, 2015. 8(3): p. 983-994.

16. Zhou, F., et al., Lattice Anharmonicity and Thermal Conductivity from Compressive Sensing of First-Principles Calculations. Physical Review Letters, 2014. 113(18): p. 185501 .

17. Eriksson, F., E. Fransson, and P. Erhart, The Hiphive Package for the Extraction of High-Order Force Constants by Machine Learning. Advanced Theory and Simulations, 2019. 2(5): p. 1800184.

18. Shay, J.L., Ternary chalcopyrite semiconductors: growth, electronic properties, and applications [by] J. L. Shay and J. H. Wernick. International series of monographs in the science of the solid state ; v. 7., ed. J.H. Wernick. 1975, Oxford, New York: Pergamon Press. 
19. Cao, Y., et al., Origin of the Distinct Thermoelectric Transport Properties of Chalcopyrite $\mathrm{ABTe}_{2}(A=C u, A g ; B=G a$, In). Advanced Functional Materials, 2020. 30(51).

20. Parker, D. and D.J. Singh, Thermoelectric properties of $\mathrm{AgGaTe}_{2}$ and related chalcopyrite structure materials. Physical Review B, 2012. 85(12): p. 125209.

21. Qiu, P., et al., Intrinsically High Thermoelectric Performance in AgInSe 2 n-Type Diamond-Like Compounds. Advanced Science, 2018. 5(3): p. 1700727.

22. Nakamura, M., et al., $\mathrm{Cd}$-free $\mathrm{Cu}(\mathrm{In}, \mathrm{Ga})(\mathrm{Se}, \mathrm{S}) 2$ thin-film solar cell with record efficiency of 23.35\%. IEEE Journal of Photovoltaics, 2019. 9(6): p. 1863-1867.

23. Wada, T., CuInSe2 and related I-III-VI2 chalcopyrite compounds for photovoltaic application. Japanese Journal of Applied Physics, 2021.

24. Thomas, S.R., et al., Recent developments in the synthesis of nanostructured chalcopyrite materials and their applications: a review. RSC advances, 2016. 6(65): p. 60643-60656.

25. Kresse, G. and J. Furthmüller, Efficient iterative schemes for ab initio total-energy calculations using a plane-wave basis set. Physical Review B, 1996. 54(16): p. 11169-11186.

26. Kresse, G. and D. Joubert, From ultrasoft pseudopotentials to the projector augmented-wave method. Physical Review B, 1999. 59(3): p. 1758-1775.

27. Grimme, S., et al., A consistent and accurate ab initio parametrization of density functional dispersion correction (DFT-D) for the 94 elements $H$-Pu. The Journal of Chemical Physics, 2010. 132(15): p. 154104.

28. Calderon, C.E., et al., The AFLOW standard for high-throughput materials science calculations. Computational Materials Science, 2015. 108: p. 233-238.

29. Park, J., Y. Xia, and V. Ozoliņš, First-principles assessment of thermoelectric properties of $\mathrm{CuFeS}_{2}$. Journal of Applied Physics, 2019. 125(12): p. 125102.

30. Plata, J.J., Posligua, V., Marquez, A. M., Fdez Sanz, J., \& Grau-Crespo, R. hiPhive Wrapper. https://github.com/NewMaterialsLab/hiPhive_wrapper_NML. GitHub Repository. 2021.

31. Bodnar, I.V., I.A. Victorov, and V.M. Dabranski, Crystal growth and investigation of $\mathrm{CuAl}_{x} \mathrm{Ga}_{1-x} \mathrm{Te}_{2}$ solid solutions. Journal of Crystal Growth, 2004. 265(1): p. 214-219.

32. Wei, S.-H. and A. Zunger, Electronic and structural anomalies in lead chalcogenides. Physical Review B, 1997. 55(20): p. 13605-13610. 
33. Zhu, Y., et al., Physical insights on the low lattice thermal conductivity of AgInSe 2 . Materials Today Physics, 2021. 19: p. 100428.

34. Rincon, C., M.L. Valerigil, and S.M. Wasim, Room-Temperature ThermalConductivity and Gruneisen-Parameter of the I-III-VI 2 Chalcopyrite Compounds. Physica Status Solidi a-Applications and Materials Science, 1995. 147(2): p. 409-415.

35. Leibfried, G. and E. Schlomann, Wärmeleitung in elektrisch isolierenden kristallen. . Nachr. Ges. Wiss. Goett. Math. -Phys. Kl. , 1954. 2(4): p. 71.

36. Bodnar, I.V., Growth and properties of CuAlSe $e_{2}$ single crystals. Inorganic Materials, 1994. 30(9): p. 1139-1141.

37. Carr, W.D., Electronic and thermal transport in copper-based chalcopyrite semiconductors for thermoelectric applications, in Department of Physics. 2016, Michigan State University: Digital Repository.

38. Berger, L.I. and V.D. Prochukhan. Ternary Diamond-Like Semiconductors / Troinye Almazopodobnye Poluprovodniki / Тройные Алмазоподобные Полупроволники. 1995.

39. Wasim, S.M. and A. Noguera, Thermal conductivity of $\mathrm{CuGaTe}$. Solid State Communications, 1987. 64(4): p. 439-442.

40. Beasley, J.D., Thermal conductivity measurements in nonlinear optical materials. OE/LASE'93: Optics, Electro-Optics, and Laser Applications in Scienceand Engineering. Vol. 1869. 1993: SPIE.

41. Charoenphakdee, A., et al., Thermal Conductivity of the Ternary Compounds: $\mathrm{AgMTe}_{2}$ and $\mathrm{AgM}_{5} \mathrm{Te}_{8}(\mathrm{M}=\mathrm{Ga}$ or In). Materials Transactions 2009. 50(7): p. 16031606.

42. Bodnar', I.V., Optical and thermal properties of $C u A l_{x} I_{1-x} T e_{2}$ solid solutions. Semiconductors, 2003. 37(11): p. 1247-1251.

43. Wasim, S.M., Thermal conductivity of ternary Compounds. physica status solidi (a), 1979. 51(1): p. K35-K40.

44. Gherouel, D., I. Gaied, and M. Amlouk, Effect of heat treatment in air on physical properties of AgInS $S_{2}$ sprayed thin films. Journal of Alloys and Compounds, 2013. 566: p. $147-155$.

45. Slack, G.A., The Thermal Conductivity of Nonmetallic Crystals, in Solid State Physics, H. Ehrenreich, F. Seitz, and D. Turnbull, Editors. 1979, Academic Press. p. $1-71$. 
46. Callaway, J., Model for Lattice Thermal Conductivity at Low Temperatures. Physical Review, 1959. 113(4): p. 1046-1051.

47. Zhu, Y., et al., Violation of the $T^{-1}$ Relationship in the Lattice Thermal Conductivity of $\mathrm{Mg}_{3} \mathrm{Sb}_{2}$ with Locally Asymmetric Vibrations. Research, 2020. 2020: p. 4589786.

48. Xia, Y., et al., Particlelike Phonon Propagation Dominates Ultralow Lattice Thermal Conductivity in Crystalline $\mathrm{Tl}_{3} \mathrm{VSe}_{4}$. Physical Review Letters, 2020. 124(6): p. 065901.

49. Makovetskaya, L.A., et al., Thermal Conductivity, Thermoelectric Power, and Thermal Expansion of CuIn $S_{2 x} \operatorname{Se}_{2(1-x)}$. physica status solidi (a), 1982. 74(1): p. K59K62.

50. Aggarwal, R.L. and T.Y. Fan, Thermal diffusivity, specific heat, thermal conductivity, coefficient of thermal expansion, and refractive-index change with temperature in AgGaSe2. Applied Optics, 2005. 44(13): p. 2673-2677.

51. Yusufu, A., et al., Effect of $\mathrm{Cu}$ Doping into the Ga Site on the Thermoelectric Properties of AgGaTe $\mathrm{A}_{2}$ with Chalcopyrite Structure. J. Jpn. Soc. Powder Powder Metallurgy, 2012. 59(4): p. 206-209. 